\title{
Reporte de caso: Bloqueo del erector de la espina en resección transuretral de próstata
}

\section{Case report: Erector spine block for transuretral prostatic resection}

\author{
María Avendaño Navarro', Ricardo Aguilar ${ }^{2, *}$ \\ Hospital México, Costa Rica. \\ 2 Centro Nacional de Rehabilitación, Costa Rica.
}

Fecha de recepción: 22 de junio de 2020 / Fecha de aceptación: 11 de octubre de 2020

\begin{abstract}
The Erector Spinae Plane (ESP) block, described in 2016 by Dr. Forero et al, was used to treat neuropathic pain and postoperative acute pain. It was described as an interfascial block in the erector spinae plane, where it manages to block both ventral and dorsal branches of the spinal nerve. Due to scarce literature on its applicability in urological endoscopic surgeries, we formulate the hypothesis that it would be an effective opioid-sparing analgesic alternative in patients undergoing TULIP. Therefore, bilateral ESP was performed in a 69-year-old patient, who underwent transurethral prostatectomy guided by laser induced (TULIP) for benign prostatic hyperplasia. The patient did not require rescue medication, reported a maximum VAS of 4/10. At 72 hours postoperatively, the patient kept a VAS of 0/10, without nausea and vomiting, with a Likert satisfaction level of 1.
\end{abstract}

Key words: Erector spine block plane, transuretral prostatic resection.

\section{RESUMEN}

El bloqueo del plano erector de la espina (ESP) fue descrito en 2016 por el Dr. Forero et al., con el afán de tratar el dolor neuropático y el dolor agudo posoperatorio. Consiste en un bloqueo interfascial en el plano del erector de la espina, en el cual se logra el bloqueo tanto de ramas ventrales como dorsales de los nervios espinales. Debido a la falta de literatura sobre su indicación en cirugías endoscópicas urológicas se formula la hipótesis de que podría considerarse como una alternativa analgésica efectiva ahorradora de opioides en pacientes sometidos a RTU-P. Por lo anterior se realizó el bloqueo ESP de manera bilateral a un paciente de 69 años, el cual fue sometido a prostatectomía transuretral guiado por láser inducido (TULIP) por hiperplasia prostática benigna. El paciente no requirió opioides de rescate, con EVA 0/10 en posoperatorio, valor que se mantuvo de la misma manera por $72 \mathrm{~h}$, tanto en reposo como en movimiento, sin la presencia de náuseas o vómitos y una escala de satisfacción de Likert de 1.

Palabras clave: Bloqueo plano erector de la espina, resección transuretral de próstata.

\section{Introducción}

E bloqueo erector de la espina fue descrito en 2016 por el Dr. Forero y colaboradores, se realizó para tratar dos casos de dolor torácico neuropático y dos de dolor agudo posoperatorio, donde se constató que disminuía significativamente el dolor. Se describió como un bloqueo de tipo interfascial con una punción a nivel de la apófisis transversa de T5, profunda al músculo erector de la espina donde se produce dispersión céfalo-caudal del anestésico, mediante el cual se logra abarcar varios dermatomas y analgesia bloqueando las ramas ventrales y dorsales de los nervios espinales[1].

Se han realizado una variedad de reporte de casos, algunos estudios controlados, que han demostrado resultados positivos de su efectividad en el manejo analgésico multimodal posoperatorio en varios tipos de cirugía como lo son pieloplastías, reconstrucción mamaria, reemplazos de cadera, hernioplastía inguinales, cirugía abdominal mayor abierta entre otros. Esto se ha realizado mediante distintos abordajes (cervical, torácico y lumbar) y técnicas (single shot, bolos intermitentes, infusión 
continua), incluso se ha utilizado como alternativa al bloqueo neuroaxial en paciente de difícil acceso con el objetivo de disminuir riesgos[2].

La efectividad analgésica del ESP se reportó en un caso de prostatectomía retropúbica radical, el bloqueo se realizó a nivel de T12 bilateralmente con resultados positivos. EVA 2/10 en descanso, 3/10 al toser por $48 \mathrm{~h}$. No se encontraron otras publicaciones similares y tampoco acerca de su aplicación en RTU-próstata[(3].

Debido a la falta de literatura de su utilización en cirugías endoscópica urológicas, se formuló la hipótesis de que el bloqueo ESP sería una alternativa analgésica efectiva ahorradora de opioides en paciente sometidos a RTU-P (resección transuretral de próstata), por lo que se plantea el siguiente reporte de caso con seguimiento por $72 \mathrm{~h}$.

\section{Reporte de caso}

Paciente masculino de 69 años, peso 73 kilos, altura 167 $\mathrm{cm}$, hipertenso, dislipidémico, en estudio por poliquistosis renal. Tratamiento crónico con amlodipino 5 mg, lovastatina 40 mg. No cuenta con alergias a medicamentos o alimentos. Sin antecedentes de consumo de alcohol, tabaco o drogas, como antecedentes quirúrgicos cuenta con un reemplazo total de cadera derecha (1998), reemplazo total de cadera izquierda (2016), revisión de reemplazo cadera derecha (2018) y cistoscopia (2018) sin complicaciones perioperatorios. Actualmente, se someterá a prostatectomía transuretral guiado por láser inducido (TULIP) por hiperplasia prostática benigna.

El paciente se premedicó con $3 \mathrm{mg}$ de midazolam intravenoso, se colocó en posición sedente. Mediante transductor convexo (GE, General Electric) se localizó la apófisis transversa a nivel de L2 parasagital y se procedió a realizar bloqueo erector de la espina en plano longitudinal céfalo-caudal con aguja 85 $\mathrm{mm}$ (Panjuk ${ }^{\circledR}$ SonoPlex), se inyectaron 20 cc de bupivacaína al 0,25\% de manera bilateral observando difusión del anestésico y elevación de músculo erector (Figura 1).

Posteriormente, como plan anestésico se realizó bloqueo espinal. Este fue llevado a cabo mediante la identificación pre-
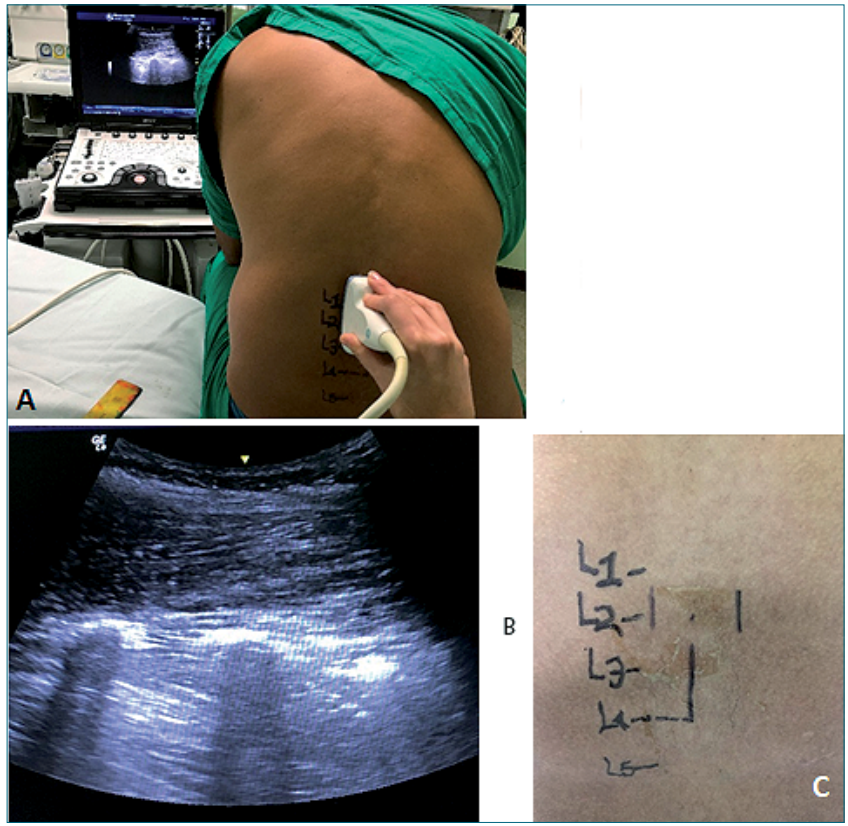

Figura 1. A) Marcas de ubicación de apófisis transversa L2; B) Marca para bloqueo neuroaxial; C) Imagen US erector de la espina.

via del espacio intervertebral ideal, por ultrasonido mediante transductor convexo, localizando L4 como el sitio óptimo que contaba con un espacio subaracnoideo a $5 \mathrm{~cm}$ de profundidad (Figura 2). Previa asepsia y antisepsia se infiltró en sitio marcado, con lidocaína al 2\% y bicarbonato 1:10. Se realizó punción con aguja punta de lápiz de $25 \mathrm{G}$ a primera intención, verificándose inmediatamente adecuado retorno de líquido cefalorraquídeo (LCR), colocándose 7,5 mg de chirocaína al 0,5\% isobárica.

Durante el período transoperatorio se realizó sedación con $2 \mathrm{mg}$ de midazolam IV y $50 \mathrm{mg}$ de propofol IV, además de 2,5 g de metamizol IV como parte del esquema analgésico multimodal. En sala de recuperación el paciente fue evaluado, contaba con una valoración de 4 en la escala de Bromage, EVA 0/10. Para manejo analgésico posoperatorio se indicó $1 \mathrm{~g}$ de aceta-
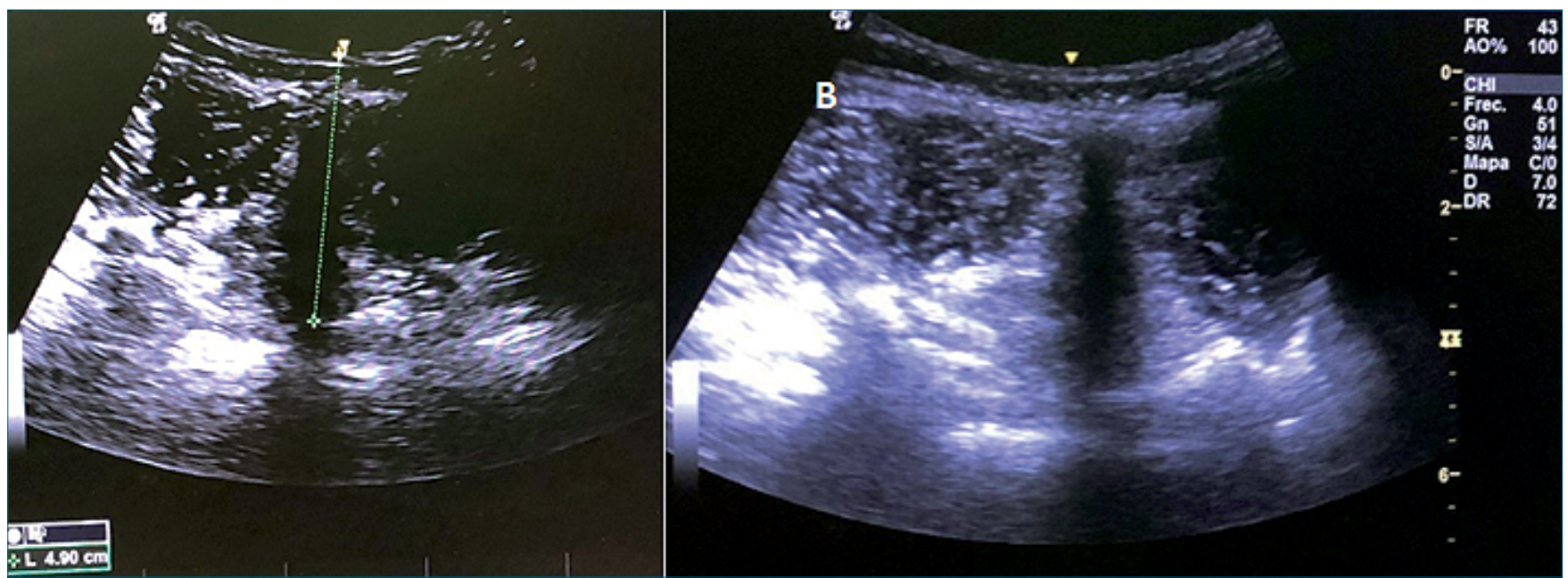

Figura 2. A) Profundidad de espacio espinal; B) Complejo anterior y posterior. 
minofén VO cada 8 h y metamizol 2,5 g IV cada 12 h con 100 mg de tramadol IV de rescate.

Durante el período posoperatorio el paciente no requirió medicación de rescate, cursó con EVA 0/10, sin náuseas o vómitos. Fue egresado a las 48 h, se siguió mediante consulta telefónica por 72 h donde el paciente siempre reportó EVA 0/10, sin náusea y vómitos, con utilización de acetaminofén $1 \mathrm{~g}$ cada 8 h como única medicación analgésica, además de un nivel de satisfacción Likert de 1 (Tabla 1).

\section{Discusión}

EI ESP fue descrito como un bloqueo interfascial por el Dr. Forero y colaboradores en el año 2016. Fue descrito para tratar el dolor neuropático, posteriormente, con los reportes de casos, los estudios cadavéricos y con resonancia magnética descubrieron su utilidad para el manejo del dolor agudo posquirúrgico, principalmente en procedimientos torácicos[1].

Es realizado a nivel de la apófisis transversa de T5 en el eje parasagital medio realizando una inyección de aproximadamente 20 cc céfalo-caudal profunda al músculo erector de la espina con difusión de múltiples dermatomas, con una sonoanatomía de fácil identificación y sin estructuras de cuidado al puncionar[1],[2].

El mecanismo por el cual brinda analgesia no está suficientemente claro. Se describió originalmente que actuaba mediante difusión de anestésico local hacia los forámenes, así lograba bloquear tanto ramas anteriores y dorsales, por lo que existía posibilidad de generar bloqueo simpático[2].[4].

Al no estar claro su mecanismo de acción, en el año 2020 se publicó un estudio el cual quería identificar la dispersión del anestésico local y así describir como lograba bloquear las ramas anteriores y posteriores, así cómo cuáles estructuras quedaban cubiertas. Este se realizó mediante la aplicación de ESP en 6 pacientes con dolor pélvico crónico a nivel de T10, por un único especialista e inmediatamente del bloqueo se llevaba a cabo la resonancia magnética. Concluyeron que se distribuía consistentemente en el espacio intercostal, forámenes neurales y músculos erectores con lo que se lograba tanto el bloqueo anterior y posterior de paredes abdominales y torácicas, pero su extensión céfalo-caudal fue muy variable. Al mismo tiempo se confirmó que su distribución puede potencialmente mejorarse por contracción del músculo erector y la presión negativa intratorácica en inspiración ayudando a llegar hasta el espacio paravertebral. Debido a que se consignó que existe posibilidad de llegar a espacio epidural, esto debe ser tomado en cuenta al utilizar volúmenes altos[4].
Actualmente, ha habido un aumento de publicaciones, especialmente de reporte de casos acerca de la utilidad y efectividad del bloqueo ESP, en distintas cirugías como nefrectomías, reemplazo de cadera, cirugía reconstructiva de mama, cirugía torácicas incluso cirugía abdominal mayor abierta y endoscópica, así como el uso de distintas técnicas como single shot, bolus intermitente e infusión continua, esto habla de la acogida del bloqueo en distintas partes del mundo y el interés hacia el mismo[2],[6].

Este bloqueo es útil en una variedad de cirugías, se puede aplicar a distintos niveles según el procedimiento y la herida quirúrgica, puede ser tanto un abordaje cervical, torácico como lumbar. En revisiones de reporte de casos aparecen el nivel torácico y la técnica de single shot como la más utilizada en población adulta, donde se constata la reducción de opioides. La mayoría de los beneficios se atribuyen a un régimen de analgesia multimodal, la duración dependerá de la concentración utilizada de anestésico local, los coadyuvantes y la técnica escogida[5].

ESP parece ser una opción segura y una alternativa a otros bloqueos como el paravertebral considerado el estándar de oro en cirugía torácica, pero el ESP es más simple de realizar y más seguro, disminuye el riesgo de neumotórax. Además, es de utilidad como alternativa al bloqueo neuroaxial al colocarse bilateralmente con menor riesgo de hematoma, punción dural y retención de orina. Se necesitan más estudios para identificar cambios a nivel motor y sensitivo al igual que la superioridad al compararse con otros bloqueos de la pared torácica[5].

En nuestro caso el paciente obtuvo una adecuada analgesia durante las $72 \mathrm{~h}$ de seguimiento, durante este período en la escala visual análogo el valor mayor que presentó fue de 4, manteniéndose generalmente en un nivel de dolor leve. Se mantuvo con un régimen de analgesia multimodal con acetaminofén y metamizol fijo, sin necesidad de requerir rescate con opioide. En este caso se aplicó el bloqueo traslapando los resultados de cirugías abdominales mayores donde el ESP ha dado buenos resultados para el manejo del dolor visceral y somático, como sucedió en este caso.

Debido a que no hay reportes de caso de su aplicación para TULIP fue que se decidió aplicar dicho bloqueo y valorar el resultado. El único antecedente que se logró consignar en la literatura fue su uso en prostatectomía radical suprapúbica[7].

Con este caso se concluye que el bloqueo ESP es una buena alternativa para prostatectomía tanto abiertas como endoscópicas, incluso podría convertirse en una indicación por su fácil aplicación, seguridad, disminución de efectos adversos y complicaciones. Deben realizarse más casos y estudios randomizados para llegar a ser una recomendación[3],[6].

\begin{tabular}{lcccl} 
& \multicolumn{2}{c}{ Tabla 1. Valoración y manejo del dolor perioperatorio. EVA: escala visual análoga } \\
\hline Posoperatorio & Likert & EVA & Náusea/vómito & Medicación \\
$12 \mathrm{~h}$ & 1 & $0 / 10$ & No & Metamizol $2,5 \mathrm{~g}$ \\
$24 \mathrm{~h}$ & 1 & No & Acetaminofén $2 \mathrm{~g}, \mathrm{metamizol} 2,5 \mathrm{~g}$ \\
$36 \mathrm{~h}$ & 1 & No & Metamizol $2,5 \mathrm{~g}$ \\
$48 \mathrm{~h}$ & 1 & $0 / 10$ & No & Acetaminofén $1 \mathrm{~g}, \mathrm{metamizol} 2,5 \mathrm{~g}$ \\
$60 \mathrm{~h}$ & $4 / 10$ & No & Acetaminofén $1 \mathrm{~g}$ \\
$72 \mathrm{~h}$ & 1 & $2 / 10$ & No & Acetaminofén $1 \mathrm{~g}$ \\
\hline
\end{tabular}




\section{Referencias}

1. The erector spinae plane block: a novel analgesic technique in thoracic neuropathic pain. Forero M, Adhikary SD, López H, Tsui C, Chin KJ. 5, s.l.: Regional Anesthesia and Pain Medicine, 2016, Vol. 41.

2. The erector spinae plane (ESP) block: A pooled review of $242 \mathrm{ca}-$ ses. Ban T, Ahtziri F, Farrukh M, Grant M, Thomas J. s.l.: Journal of Clinical Anesthesia, 2019, Vol. 53.

3. Ultrasound guide low thoracic erector spinae plane block for postoperative analgesia in radical retropubic prostatectomy, a new indication. Serkan T, Ozgur S. s.l.: Journal of Clinical Anesthesia, 2018, Vol. 47.

4. A magnetic resonance imaging study of local anesthetic spread in patients receiving an erector spinae plane block . Schwartzmann, A, Peng P, Antunez M, Alcarraz P, González X, Forero M. s.I.: Canadian Journal of Anesthesia, 2020.

5. Bilateral continuous erector spinae plane block contributes to effective postoperative analgesia after major open abdominal surgery: case report . Restrepo C, Chin K, Suarez P, Díaz A. s.I.: Anesthesia \& Analgesia, 2017, Vol. 19.

6. Erector spinae plane block for different laparascopic abdominal surgeries: case series. Serkan T, Onur S, Mahmut S. s.I.: Case reports in anesthesiology, 2018.

7. Multimodal analgesic approach incorporating paravertebral blocks for open radical retropubic prostatectomy: a randomized double - blind placebo- controlled study. Jaques E, Taras P, Joel B. s.I.: Canadian Journal of Anesthesia, 2010, Vol. 58. 